\title{
A Nonparametric Revealed Preference Approach to Measuring the Value of Environmental Quality
}

\author{
Laura Blow ${ }^{1,2}$. Richard Blundell ${ }^{2,3}$
}

Published online: 26 February 2018

(C) The Author(s) 2018. This article is an open access publication

\begin{abstract}
We develop an approach to valuing non-market goods using nonparametric revealed preference analysis. We show how nonparametric methods can also be used to bound the welfare effects of changes in the provision of a non-market good. Our main context is one in which the non-market good affects the marginal utility of consuming a related market good. This can also be framed as a shift in the taste for, or quality of, the market good. A systematic approach for incorporating quality/taste variation into a revealed preference framework for heterogeneous consumers is developed. This enables the recovery of the minimal variation in quality required to rationalise observed choices of related market goods. The variation in quality appears as a adjustment to the price for related market goods which then allows a revealed preference approach to bounding compensation measures of welfare effects to be applied.
\end{abstract}

Keywords Revealed preference · Bounds · Public good valuation · Inequality restrictions · Semiparametric regression · Changing tastes

Guest Editor: V. Kerry Smith.

We would like to thank Kerry Smith, two anonymous commentators and participants at the conference for comments on an earlier draft. The authors also gratefully acknowledge financial support from the Economic and Social Research Council Centre for the Microeconomic Analysis of Public Policy at IFS.

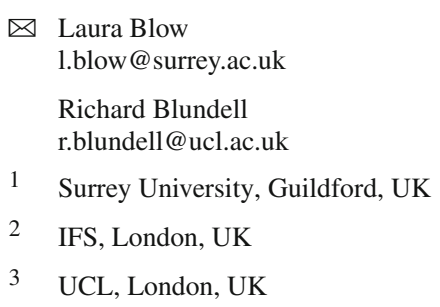




\section{Introduction}

In this article, we use a common method of valuing non-market environmental goods whereby information about the valuation of a public good is extracted from its relationship with a marketed good. Existing specific examples of this method include household production theory where the household combines market goods with an environmental good to produce the service from which they ultimately derive utility, and the assumption of weak complementarity or substitutability between the surrogate market good and the environmental good.

We develop a new approach to this general method that uses nonparametric revealed preference analysis. We believe this could be a helpful approach for two main reasons: firstly it does not rely on particular functional forms for demand which have the potential to influence heavily the valuation estimates; secondly the marginal valuation of changes in the quality of the environmental good are allowed to differ in a very flexible way across consumers. This second point means that this approach could also be very flexible and useful from a benefit transfer point of view, provided we have information on the distribution of relevant characteristics for the transfer population. We now discuss both these points in a little more detail.

Revealed preference theory was pioneered by Samuelson (1948). At that time, the traditional approach to demand theory (still widely used today) was parametric; a particular functional form for demand functions was estimated and then consideration was given to the restrictions those functions must satisfy if they had been generated by utility maximisation. One problem endemic to parametric demand estimation is that predictions can be heavily dependent on the functional form used to estimate demands. Indeed this has been discussed by Crooker and Kling (2000) in the case of environmental valuation. This has potential to be a significant issue in applications using weak complementarity, for example, since the demand curve for the weakly complementary market good must be extrapolated to zero.

Nonparametric revealed preference theory generates elegant nonparametric tests that can be used to assess whether data on observed consumer choices is consistent with having been generated by utility maximisation without having to impose a particular functional form on preferences. Building on the work of Samuelson (1948), Houthakker (1950) and Afriat (1967), Varian $(1982,1983)$ solved or simplified many of the most important computational aspects of revealed preference. He also showed that if the data satisfies revealed preference tests of maximising behaviour, then revealed preference theory can be used to recover information about the utility function and to forecast choices at new budgets or prices.

Blundell et al. $(2003,2008)$ developed a method for choosing a sequence of total expenditures that maximize the power of tests of generalized axiom of revealed preference (GARP) with respect to a given preference ordering. They term this the sequential maximum power (SMP) path and present some simulation evidence showing that these GARP tests have considerable power against some key alternatives. From this idea it is possible to develop a method of generating best bounds on the welfare costs of relative price or tax changes. In particular, Blundell et al. obtain the tightest upper and lower bounds for indifference curves passing through any chosen point in the commodity space. The tightness of these bounds depends on the closeness of the new prices to the sets of previously observed prices and the restrictions placed on cross-price effects.

If environmental quality was a marketed good (bad) then the welfare gain from reducing the level of the environmental quality across the income distribution could be measured nonparametrically using this nonparametric revealed preference bounds analysis. Crooker and Kling (2000) outline the use of the Varian bounds approach for this case in the particular 
situation where observed data are generated under fixed nominal income and a varying price for the environmental good.

The more usual case is where the environmental good is not marketed. Typically we also cannot perfectly observe and quantify an environmental good in the same way as a market good; we have some indicators of water quality, say, but that it is a different situation from being able to say the household has consumed a certain number of apples. Suppose first that its level can be measured. In that case we can think of the environmental good as being a special case of a rationed good. Hicks (1940) and Rothbarth (1941) and more recently Neary and Roberts (1980) discuss the question of how to deal with rationed goods in economic problems, and in particular how to price goods when the consumer is free to purchase goods in some markets, but forced to purchase certain levels of other goods in other markets. They show how the properties of demands under these circumstances can be expressed in terms of unrationed demands by allowing free choice over all goods but replacing the observed market prices with a vector of 'virtual' prices or 'support' prices. These support prices are such that this unrationed choice would generate exactly the same demand vector as the one generated by the observed prices under the rationing constraint. Under certain conditions, the support prices can be used to recover the welfare impact of changing the level of the rationed good, in this case the level of the environmental good. The required conditions rule out the rationed good, or environmental good, entering individual welfare in any other way than in combination with the unrationed goods. If these conditions are not met then the part of the welfare cost is identified. The stated preference method attempts to overcome this identification problem by eliciting values for the non-marketed good. Whilst acknowledging this issue, we will focus solely on use value of the environmental good.

Where the environmental good is not perfectly observed we develop on from the procedure for the rationed good case by noting that changes in the value of the indicators of the quality of the environmental good will still change the marginal utility of related marketed goods. This will appear like a change in the tastes for those related goods and consequently will show up as rejections of the nonparametric revealed preference conditions. Following Adams et al. (2015) we thus allow for local perturbations to marginal utility to describe the degree of quality change. In ABBC, a method is developed for use with consumer survey data for finding the minimal shift in marginal utility that corrects nonparametric revealed preference rejections. This shift is allowed to differ in a very flexible way across consumers. To implement the approach, a minimum distance method is used in order to estimate perturbed demands that are local to each income percentile and data period. The minimum is taken subject to the revealed preference conditions. We can also relate the observable indicators of environmental quality to the utility adjustments we find, particularly if we have multiple observations of quality change. This would aid benefit transfer calculations.

Here we assess this approach as a method for valuing the welfare cost of changes in an environmental good. The method can be used with consumer survey data for finding bounds on the shift in marginal utility caused by the change in the environmental good where, again, this shift is allowed to differ in a very flexible way across consumers. This allows us to assess the importance of changes in environmental quality and how this differs across the income distribution. We also show that, once the perturbations to marginal utility are estimated, these form the basis to using the same nonparametric revealed preference methods to bounding the welfare effects of a change to environmental quality.

The ABBC marginal utility shift model can be thought of a constant preference model where observed prices are replaced with taste-adjusted prices. In the environmental good context we would think of this a quality-or hedonic - adjustment to prices. Of course, the idea of translating the welfare effects from changes in unmarketed goods to changes in the 
prices of some marketed goods is hardly new (Gorman 1956; Lancaster 1966; Fisher and Shell 1971; Muellbauer 1975; Willig 1978). Hedonic pricing is a method that is already well documented in connection to environmental valuation (Bockstael and McConnell 1993; Smith and Banzhaf 2004, 2007; Palmquist 2005) and some of the techniques for conceptualising this method are very similar to ideas used in the equivalence scale literature (Lewbel 1985; Pollak 1989; Blackorby and Donaldson 1993).

With the estimate of the price adjustment in hand, this then allows the welfare consequences of an environmental quality change to be calculated in a standard way, for example a compensating variation. Blundell et al. (2003) show how compensating variation can be bounded using a nonparametric approach, and we outline their approach in the present context. The advantage of this method is that is gives bounds on welfare measure that are consistent with any well behaved preferences that could have rationalised the underlying data, thus avoiding errors arising from erroneous functional assumptions.

In what follows we first review the key ideas and set up our general notation in Sect. 2. In Sect. 3 we give a brief explanation of nonparametric revealed preference theory. We then, in Sect. 4, examine the use of nonparametric revealed preference for the valuation of nonmarketed environmental goods, distinguishing between the case where the environmental good (bad) can be measured like a normal market good from the case where we observe indicators of environmental quality. Section 5 develops a general method for bounding the compensating variation in the unobserved quality case. Section 6 describes our empirical approach and Sect. 7 concludes.

\section{Non-marketed Environmental Goods}

One of the main challenges of environmental valuation is, of course, that environmental goods are not marketed and so we do not observe prices. Most valuation techniques that rely on observed consumer behaviour use some method of calculating the implicit price of the environmental good by hypothesising some relationship between the environmental good and one or more marketed commodities consumed by the household. Although this is well known, we take some time below to set up the general problem as this will help later with describing our proposed approach.

Suppose there are $T+1$ periods, $t=0, \ldots, T$ in which a consumer is choosing over $K+1$ market goods and services, $k=0, \ldots, K$ with an exogenously set level of a public or environmental good $q_{t}$ that is also a determinant of their welfare. We can think of the environmental public good as being a special case of a rationed good. Its market price is zero, and the consumer can only consume $q_{t}$.

As described in the introduction Neary and Roberts (1980) show how the properties of demands under rationing can be expressed in terms of unrationed demands by allowing free choice over all goods but replacing the observed market prices with a vector of 'virtual' prices or 'support' prices. ${ }^{1}$ These support prices are such that this unrationed choice would generate exactly the same demand vector as the one generated by the observed prices under the rationing constraint. Neary and Roberts (1980) show that convexity, continuity and strict monotonicity of the consumer's preferences are sufficient to ensure that there always exists a set of strictly positive support prices consistent with any set of demands.

\footnotetext{
1 See also Chavas (1984) for a related theory of mixed demand systems where some goods have an exogenous price which then determines consumer demand and some goods have an exogenously fixed supply which then determines market price (given consumer preferences).
} 
In the present context, the consumer's problem is:

$$
\max _{\mathbf{x}, q} U(\mathbf{x}, q) \text { subject to } \mathbf{p}_{t}^{\prime} \mathbf{x} \leq y_{t} \text { and } q=q_{t}
$$

where $y_{t}$ denotes the available budget in period $t$ and the price vector is $\mathbf{p}_{t}$. Assuming non-satiation and, for simplicity, that the consumer buys some of all goods, the first order conditions are

$$
\left[\begin{array}{c}
U_{\mathbf{x}}(\mathbf{x}, q) \\
U_{q}(\mathbf{x}, q)
\end{array}\right]=\lambda_{t}\left[\begin{array}{c}
\mathbf{p}_{t} \\
\frac{\mu_{t}}{\lambda_{t}}
\end{array}\right]
$$

The scalar $\lambda_{t}$ is the multiplier on the budget constraint (marginal utility of income) and $\mu_{t}$ is multiplier on the rationing constraint on the public good. Here, clearly, the support prices for the market goods are simply the market prices. The virtual, or shadow, price for the public good is $\mu_{t} / \lambda_{t}$. We will denote this by $\pi_{t}^{m}=\pi(y, \mathbf{p}, q)$ (the superscript $m$ indicates Marshallian).

The support prices are such that the outcome of the rationed model is identical to the outcome of the unrationed choice generated by

$$
\max _{\mathbf{x}, q} U(\mathbf{x}, q) \text { subject to } \mathbf{p}_{t}^{\prime} \mathbf{x}+\pi_{t} q \leq y_{t}+\pi_{t} q
$$

Likewise, looking at the cost function we see:

$$
c(u, \mathbf{p}, q)=\min _{x} \mathbf{p}_{t}^{\prime} \mathbf{x} \text { subject to } U(\mathbf{x}, q) \geq u \text { and } q=q_{t}
$$

The envelope theorem gives us the following relationships (where $\gamma$ is the multiplier on the utility constraint):

$$
\begin{aligned}
& c_{u}(u, \mathbf{p}, q)=\gamma(u, \mathbf{p}, q) \\
& c_{\mathbf{p}}(u, \mathbf{p}, q)=\mathbf{x}^{h}(u, \mathbf{p}, q) \\
& c_{q}(u, \mathbf{p}, q)=-\gamma U_{q}\left(\mathbf{x}^{h}, q\right)
\end{aligned}
$$

We can think of Eq. (1) as defining a shadow price $\pi_{t}^{h}=\pi^{h}(u, \mathbf{p}, q)$ for $q$ (the superscript $h$ indicates Hicksian).:

$$
\pi^{h}(u, \mathbf{p}, q)=-c_{q}(u, \mathbf{p}, q)=\gamma U_{q}\left(\mathbf{x}^{h}, q\right)
$$

Therefore we can now write an unconstrained cost function giving identical outcomes to the constrained one as

$$
c\left(u, \mathbf{p}, \pi^{h}\right)=\min _{x} \mathbf{p}_{t}^{\prime} \mathbf{x}+\pi^{h} q \text { subject to } U(\mathbf{x}, q) \geq u
$$

and so

$$
c\left(u, \mathbf{p}, \pi^{h}(u, \mathbf{p}, q)\right)=c(u, \mathbf{p}, q)+\pi^{h}(u, \mathbf{p}, q) q
$$

Note that here $\pi^{m}(c(u, \mathbf{p}, q), \mathbf{p}, q)=\pi^{h}(u, \mathbf{p}, q)$. Unless the distinction is necessary, we will simply call this $\pi$ from now on to avoid cluttered notation.

The problem we face is to calculate some welfare measure from a change in the level of the public good-suppose there are two periods and $q$ changes from $q_{0}$ to $q_{1}$, with prices of market goods remaining unchanged $\mathbf{p}_{0}=\mathbf{p}_{1}=\mathbf{p}$. Then the compensating variation is

$$
C V_{0,1}=c\left(u_{0}, \mathbf{p}, q_{1}\right)-c\left(u_{0}, \mathbf{p}, q_{0}\right)
$$


and the equivalent variation is

$$
E V_{0,1}=c\left(u_{1}, \mathbf{p}, q_{1}\right)-c\left(u_{1}, \mathbf{p}, q_{0}\right)
$$

If we knew $\pi(u, \mathbf{p}, q)$ then

$$
c\left(u, \mathbf{p}, q_{0}\right)-c\left(u, \mathbf{p}, q_{1}\right)=\int_{q_{0}}^{q_{1}} \pi(u, \mathbf{p}, q) d q
$$

But obviously we do not observe $\pi(u, \mathbf{p}, q)$. The challenge is to find ways to learn about this function, and the main approaches do this by supposing there is some relationship in the utility function between $q$ and one or more market good. One of the earliest examples is Maler's idea of perfect substitutability between the environmental good $q$ and a market good, which we will denote as $x^{0}$, in the household production of some final service; an example would be a defensive expenditure model where a water purifier is a perfect substitute for environmental water quality in the production of clean water for the household. The reason that Maler's model provides a solution is that if $U(\mathbf{x}, q)=U\left(\mathbf{x}^{K}, F\left(x^{0}, q\right)\right)$ (where $\mathbf{x}^{K}$ denotes all goods other than $\left.x^{0}\right)$ and $F\left(x^{0}, q\right)=F\left(x^{0}+\tau q\right)$ then we simply have $\pi=\tau p^{0}$ and, for example, $C V_{0,1}=\tau p^{0}\left(q_{0}-q_{1}\right)$. Clearly this would also require knowledge of $\tau$. Without perfect substitutability, this simple result no longer holds and knowledge of the production function is required.

A related application of the household production model is the hedonic pricing approach. Here it is supposed, for example, that the household produces housing services from their accommodation and local public goods. If the quality of public goods varies across locations then this can be expected to be reflected in prices for otherwise equivalent accommodation. A regression of house prices on local characteristics is then used to infer households' valuations of public goods. The form of regression depends on the form hypothesised for the household production function, the most common approach being the linear characteristics model.

An interesting alternative approach sometimes used in environmental applications is to assume that the surrogate market good is a weak complement to the quality of the environmental input, which means that if the consumer does not consume the surrogate good he receives no benefit from the environmental good (Bockstael and McConnell 1993; Smith and Banzhaf 2004, 2007; Palmquist 2005). This allows the researcher to measure something like compensating variation due to a change in environmental quality as the difference in area between two compensated demand curves (one for the demand of the surrogate good under initial quality and one for demand under the new quality level) from the observed market price to price that induces zero demand. Herriges et al. (2004) present a method for extending this methodology without assuming weak complementarity. Some extra assumptions can be made ${ }^{2}$ in order to allow Marshallian demand curves to be used instead of compensated demand curves to approximate the welfare costs.

A related, but much less common, approach is to assume weak substitutability between a market good and environmental quality (Feenberg and Mills 1980; Smith et al. 2010). This means that there will now be a level of consumption of the market good above which improvements in the environment no longer benefit the consumer.

As discussed in the introduction, these parametric measures of environmental valuation can be heavily dependent on the functional form used to estimate demands or price equations. This is the motivation for our approach in this chapter in which we aim to explore how we could use revealed preference analysis [in the Samuelson (1948), Houthakker (1950) and Afriat

2 The Willig condition. 
(1967) sense] to learn about $\pi(u, \mathbf{p}, q)$. In the next section we give a brief overview of revealed preference theory.

\section{Nonparametric Revealed Preference Theory}

Revealed preference theory builds on the simple idea that given a vector of prices and choices, $\mathbf{p}_{t}$ and $\mathbf{x}_{t}$, at time $t$, then we can say that the choice of $\mathbf{x}_{t}$ over another bundle $\mathbf{x}$ that the consumer could also have afforded means that the consumer has revealed a preference for $\mathbf{x}_{t}$ over $\mathbf{x}$.

As we will be using a revealed preference approach throughout this chapter, we will briefly describe the salient points. Following Varian (1982) we set out the following definitions

Definition 1 A utility function $U(\mathbf{x})$ rationalises the dataset $\left(\mathbf{p}_{t}, \mathbf{x}_{t}\right) t=0, \ldots, T$ if $U\left(\mathbf{x}_{t}\right) \geq U(\mathbf{x})$ for all $\mathbf{x}$ such that $\mathbf{p}_{t}^{\prime} \mathbf{x}_{t} \geq \mathbf{p}_{t}^{\prime} \mathbf{x}$, for $t=0, \ldots, T$.

Definition 2 An observation $\mathbf{x}_{t}$ is directly revealed preferred to an observation $\mathbf{x}$, written $\mathbf{x}_{t} R^{0} \mathbf{x}$, if $\mathbf{p}_{t}^{\prime} \mathbf{x}_{t} \geq \mathbf{p}_{t}^{\prime} \mathbf{x}$. An observation $\mathbf{x}_{t}$ is revealed preferred to an observation $\mathbf{x}$, written $\mathbf{x}_{t} R \mathbf{x}$, if there is some sequence of observations $\left(\mathbf{x}_{j}, \mathbf{x}_{k}, \ldots, \mathbf{x}_{l}\right)$ such that $\mathbf{x}_{t} R^{0} \mathbf{x}_{j}, \mathbf{x}_{j} R^{0} \mathbf{x}_{k}$, $\ldots, \mathbf{x}_{l} R^{0} \mathbf{x}$. In this case we say $R$ is the transitive closure of the relation $R^{0}$.

Definition 3 An observation $\mathbf{x}_{t}$ is directly revealed strictly preferred to an observation $\mathbf{x}$, written $\mathbf{x}_{t} P^{0} \mathbf{x}$, if $\mathbf{p}_{t}^{\prime} \mathbf{x}_{t}>\mathbf{p}_{t}^{\prime} \mathbf{x}$. An observation $\mathbf{x}_{t}$ is revealed strictly preferred to an observation $\mathbf{x}$, written $\mathbf{x}_{t} P \mathbf{x}$, if there is some sequence of observations $\left(\mathbf{x}_{j}, \mathbf{x}_{k}, \ldots, \mathbf{x}_{l}\right)$ such that $\mathbf{x}_{t} R^{0} \mathbf{x}_{j}, \mathbf{x}_{j} R^{0} \mathbf{x}_{k}, \ldots, \mathbf{x}_{l} R^{0} \mathbf{x}$ and at least one of the inequalities is strict..

Definition 4 Data satisfy the Generalised Axiom of Revealed Preference (GARP) if $\mathbf{x}_{t} R \mathbf{x}_{s} \Rightarrow \mathbf{p}_{s}^{\prime} \mathbf{x}_{s} \leq \mathbf{p}_{s}^{\prime} \mathbf{x}_{t}$. Equivalently, the data satisfy GARP if $\mathbf{x}_{t} R \mathbf{x}_{s}$ implies not $\mathbf{x}_{s} P^{0} \mathbf{x}_{t}$.

It turns out that the GARP condition is an observable consequence of constrained rational choice, and that it is also a sufficient condition for it. If a dataset is consistent with GARP there exists a utility function which can rationalise it and it is also consistent with the costminimisation model of behaviour. This is summarised by the following theorem due to Afriat (1967), Erwin Diewert (1973) and Varian (1982).

Afriat's Theorem ${ }^{3}$ The following statements are equivalent:

(1) There exists a non-satiated utility function which rationalises the dataset $\left(\mathbf{p}_{t}, \mathbf{x}_{t}\right) \quad t=$ $1, \ldots, T$.

(2) There exist numbers $U_{s}, U_{t}, \lambda_{t} \geq 0$, for $s, t=0, \ldots, T$ such that

$$
U_{s} \leq U_{t}+\lambda_{t} \mathbf{p}_{t}^{\prime}\left(\mathbf{x}_{s}-\mathbf{x}_{t}\right)
$$

(3) The data satisfy the Generalised Axiom of Revealed Preference.

(4) There exists a continuous, concave, strictly increasing utility function which rationalises the data.

3 This Theorem with a different, but equivalent, version of condition (3) was proved by Afriat (1967). Erwin Diewert (1973) also provided a proof which omits condition (3). Varian (1982) provides a proof which swaps Afriat's verison of (3) for GARP. 


\section{A Revealed Preference Approach To Environmental Valuation}

\subsection{Perfectly Quantifiable Levels of Environmental Goods}

The way that the revealed preference approach often proceeds is finding, for the model of interest, equations that are analogous to the Afriat inequalities of the classic revealed preference results. The analysis we will propose here, therefore, will be made clearer by a brief explanation of the derivation of these inequalities. The derivation of the Afriat inequalities is easy under the standard assumption of concavity. ${ }^{4}$ Concavity of the utility function $U(\mathbf{x})$ immediately gives that for any pair of observed choices $\mathbf{x}_{t}$ and $\mathbf{x}_{S}$

$$
U\left(\mathbf{x}_{s}\right) \leq U\left(\mathbf{x}_{t}\right)+U_{\mathbf{x}}\left(\mathbf{x}_{t}\right)\left(\mathbf{x}_{s}-\mathbf{x}_{t}\right)
$$

where $U_{\mathbf{x}}\left(\mathbf{x}_{t}\right)$ denotes the vector of derivatives of $U\left(\mathbf{x}_{t}\right)$ with respect to $x_{t}^{k}$.

Substituting in first order conditions from utility maximisation gives:

$$
U\left(\mathbf{x}_{s}\right) \leq U\left(\mathbf{x}_{t}\right)+\lambda_{t} \mathbf{p}_{t}^{\prime}\left(\mathbf{x}_{s}-\mathbf{x}_{t}\right)
$$

Our task here is to find $\left\{\pi_{t}\right\}_{t=1, \ldots, T}$ so that the augmented data

$$
\left\{\mathbf{p}_{t}, \pi_{t} ; \mathbf{x}_{t}, q_{t}\right\}_{t=0, \ldots, T}
$$

passes GARP.

We can do this by formulating the problem as finding the solution to a set of Afriat-like inequalities. Concavity of the utility function $U(\mathbf{x}, q)$ gives

$$
U\left(\mathbf{x}_{s}, q_{s}\right) \leq U\left(\mathbf{x}_{t}, q_{t}\right)+U_{\mathbf{x}}\left(\mathbf{x}_{t}, q_{t}\right)\left(\mathbf{x}_{s}-\mathbf{x}_{t}\right)+U_{q}\left(\mathbf{x}_{t}, q_{t}\right)\left(q_{s}-q_{t}\right)
$$

Substituting in the first order conditions gives

$$
U\left(\mathbf{x}_{s}\right) \leq U\left(\mathbf{x}_{t}\right)+\lambda_{t} \mathbf{p}_{t}^{\prime}\left(\mathbf{x}_{s}-\mathbf{x}_{t}\right)+\lambda_{t} \pi_{t}\left(q_{s}-q_{t}\right)
$$

Thus we are asking whether there exist numbers $U_{s}, U_{t}, \lambda_{t}>0, \pi_{t}$ for $s, t=0, \ldots, T$ such that

$$
U_{s} \leq U_{t}+\lambda_{t} \mathbf{p}_{t}^{\prime}\left(\mathbf{x}_{s}-\mathbf{x}_{t}\right)+\lambda_{t} \pi_{t}\left(q_{s}-q_{t}\right)
$$

We will assume that the environmental good is described as a "good" (i.e. water quality rather than water pollution) so that we are looking for $\pi_{t} \geq 0$. This is simply a linear programming problem since we can rewrite the problem as finding whether there exist numbers $U_{s}, U_{t}, \lambda_{t}>0, \varphi_{t}>0$ for $s, t=0, \ldots, T$ such that

$$
U_{s} \leq U_{t}+\lambda_{t} \mathbf{p}_{t}^{\prime}\left(\mathbf{x}_{s}-\mathbf{x}_{t}\right)+\varphi_{t}\left(q_{s}-q_{t}\right)
$$

and then set $\pi_{t}=\varphi_{t} / \lambda_{t}$.

Varian (1988) explores a similar case to this where the consumer is choosing among $K+1$ market goods but the researcher does not observe the price for good $K+1$. He shows that a price $p_{t}^{K+1}$ can always be constructed so that the entire dataset $\left\{p_{t}^{k} ; x_{t}^{k}\right\}_{t=0, \ldots, T, k=0, \ldots, K+1}$ passes GARP simply by choosing high enough prices $p_{t}^{K+1}$ so that expenditure on good $K+1$ always dominates the revealed preference comparison. This can easily be seen by considering Eq. (2). If $\left(q_{s}-q_{t}\right)<0$ we can always choose a high enough $\pi_{t}$ so that $\mathbf{p}_{t}^{\prime}\left(\mathbf{x}_{s}-\mathbf{x}_{t}\right)+$ $\pi_{t}\left(q_{s}-q_{t}\right)<0$, i.e. $\left(\mathbf{x}_{t}, q_{t}\right) R^{0}\left(\mathbf{x}_{s}, q_{s}\right)$. If $\left(q_{s}-q_{t}\right)>0$ we can always choose a high

4 Concavity is not necessary but makes the derivation less complicated, see Varian (1982) or Fostel et al. (2004). 
enough $\pi_{t}$ so that $\mathbf{p}_{t}^{\prime}\left(\mathbf{x}_{s}-\mathbf{x}_{t}\right)+\pi_{t}\left(q_{s}-q_{t}\right)>0$, i.e. $\left(\mathbf{x}_{t}, q_{t}\right) \neg R^{0}\left(\mathbf{x}_{s}, q_{s}\right)$. In this way the revealed preference relation is dictated entirely by $q_{t}$ such that $\left(\mathbf{x}_{t}, q_{t}\right) R^{0}\left(\mathbf{x}_{s}, q_{s}\right) \Longleftrightarrow q_{t}>$ $q_{s}$, so that $\left(\mathbf{x}_{t}, q_{t}\right) R^{0}\left(\mathbf{x}_{s}, q_{s}\right) \Rightarrow\left(\mathbf{x}_{s}, q_{s}\right) \neg R^{0}\left(\mathbf{x}_{t}, q_{t}\right)$ and we will never observe a violation of GARP.

The question then becomes how can we put a more meaningful bound on the missing prices, in our case the shadow price $\pi_{t}$. For example one possibility would be to choose prices that minimise total spending on the environmental good:

$$
\begin{aligned}
& \min \sum_{t=0}^{T} \frac{\varphi_{t}}{\lambda_{t}} q_{t} \\
& \text { subject to } \\
& U_{s} \leq U_{t}+\lambda_{t} \mathbf{p}_{t}^{\prime}\left(\mathbf{x}_{s}-\mathbf{x}_{t}\right)+\varphi_{t}\left(q_{s}-q_{t}\right)
\end{aligned}
$$

\subsection{Indicators of Environmental Quality}

In the more usual case we might think that the environmental good cannot be given a quantity in the same was as a market good, but that we have indicators of environmental quality that tell us that $q$ has changed. When there is a relationship between $q$ and the marginal utility of a market good then we may be able to use this to infer information about the value of the environmental good to consumers. Instead of being able to find the implicit price $\pi_{t}$ directly as in the previous section, we now think of changes in the quality of the environmental good as affecting the utility obtained from the surrogate market good. This in turn allows us to estimate a shadow price for the market good that adds the to the observable market price a term reflecting the valuation of the quality of the environment. Readers may recognise this as an application of the hedonic pricing discussed above. Normally the hedonic approach relates the observed market price of a good to its underlying characteristics using observable variation in the product (e.g. the specification of a computer or a car). There are different approaches: for example the characteristics model (Gorman 1956; Lancaster 1966) views a good as a bundle of underlying attributes that the consumer has preferences over; the repackaging model (Fisher and Shell 1971) treats an increase in quality of a good as being like getting more of the good at the baseline quality (ten trips to the beach this summer are as good as eleven trips last summer). In empirical applications (of which there are also many non-environmental kinds) these models end up being estimated in a rather similar way, in that researchers typically also model quality change in the repackaging model as a function of observable characteristics. Hence market prices of the good are modelled as an assumed function of underlying characteristics and some type of hedonic price equation is estimated.

What we do here is slightly different in that now the price of the market good that is functionally related to environmental quality does not include the utility effect of this environmental good. When environmental quality changes for a set of consumers, we can expect this lead to rejections of the nonparametric revealed preference conditions for their observed choices. Following Adams et al. (2015), treating the quality change as a local perturbation to the marginal utility from the surrogate market good, (which translates to a change in its shadow price) allows us to use the revealed preference approach to find the minimal shift in marginal utility that corrects nonparametric revealed preference rejections. Furthermore we can relate the observable indicators of environmental quality to the utility adjustments we find, particularly if we have multiple observations of quality change. This would aid benefit transfer calculations. 
Let $x_{t}^{0}$ be the good that is affected by quality change (and denote all other goods by vector $\mathbf{x}^{K}$ ). Suppose we normalise the utility function at some level of the environmental good, say the initial level, $U\left(x^{0}, \mathbf{x}^{K}, q_{0}\right)$. We will drop the $q_{0}$ argument for ease and simply write $U\left(x^{0}, \mathbf{x}^{K}\right)$. The aim is then to be able to write the utility function at any other level of $q, U\left(x^{0}, \mathbf{x}^{K}, q_{t}\right)$, as a variant of $U\left(x^{0}, \mathbf{x}^{K}\right)$ but where the marginal utility obtained from $x^{0}$ is transformed.

The ABBC model uses the following form

$$
U\left(x^{0}, \mathbf{x}^{K}, q_{t}\right)=U\left(x_{t}^{0}, \mathbf{x}_{t}^{K}\right)+\alpha_{t} x_{t}^{0}
$$

Thus $\alpha_{t}$ is a change to the marginal utility from good 0 . This is allowed to be very general in that $\alpha_{t}$ can vary with individual characteristics such as income over time in a quite general form. In this way, the model can be thought of as an approximation to other commonly used models. For example, taking an approximation to the repackaging model $U\left(x^{0}, \mathbf{x}^{K}, q_{t}\right)=$ $U\left(a_{t} x_{t}^{0}, \mathbf{x}_{t}^{K}\right)$ gives

$$
U\left(a_{t} x_{t}^{0}, \mathbf{x}_{t}^{K}\right) \approx U\left(x_{t}^{0}, \mathbf{x}_{t}^{K}\right)+\frac{\partial U\left(x_{t}^{0}, \mathbf{x}_{t}^{K}\right)}{\partial x_{t}^{0}} x_{t}^{0}\left(a_{t}-1\right)
$$

Therefore we can think of

$$
\alpha_{t} \approx \frac{\partial U\left(x_{t}^{0}, \mathbf{x}_{t}^{K}\right)}{\partial x_{t}^{0}}\left(a_{t}-1\right)
$$

with, as mentioned, $\alpha_{t}$ being allowed to vary quite generally. There are other similarly motivated models such as the translating model $U\left(x_{t}^{0}+\beta_{t}, \mathbf{x}_{t}^{K}\right)$ which is identical to the demographic translating model of Pollak and Wales (1980) (just as the repackaging model is identical to demographic scaling), or the cross-product repackaging model.

The first order conditions from utility maximisation in the ABBC model are

$$
\begin{aligned}
& \frac{\partial\left(U\left(x_{t}^{0}, \mathbf{x}_{t}^{K}\right)+\alpha_{t} x_{t}^{0}\right)}{\partial x_{t}^{0}}=\frac{\partial U\left(x_{t}^{0}, \mathbf{x}_{t}^{K}\right)}{\partial x_{t}^{0}}+\alpha_{t}=\lambda_{t} p_{t}^{0} \\
& \frac{\partial\left(U\left(x_{t}^{0}, \mathbf{x}_{t}^{K}\right)+\alpha_{t} x_{t}^{0}\right)}{\partial x_{t}^{k}}=\frac{\partial U\left(x_{t}^{0}, \mathbf{x}_{t}^{K}\right)}{\partial x_{t}^{k}}=\lambda_{t} p_{t}^{k} \quad \forall k \neq 0
\end{aligned}
$$

and so this can be thought of as maximising the base utility $U\left(x_{t}^{0}, \mathbf{x}_{t}^{K}\right)$ with the price of good $x_{t}^{0}$ adjusted to $\left(p_{t}^{0}-\alpha_{t} / \lambda_{t}\right)$.

The Afriat-type conditions become

$$
\begin{aligned}
U_{s} & \leq U_{t}+\lambda_{t} \mathbf{p}_{t}^{K^{\prime}}\left(\mathbf{x}_{s}^{K}-\mathbf{x}_{t}^{K}\right)+\lambda_{t}\left(p_{t}^{0}-\frac{\alpha_{t}}{\lambda_{t}}\right)\left(x_{s}^{0}-x_{t}^{0}\right) \\
& =U_{t}+\lambda_{t} \mathbf{p}_{t}^{\prime}\left(\mathbf{x}_{s}-\mathbf{x}_{t}\right)-\alpha_{t}\left(x_{s}^{0}-x_{t}^{0}\right)
\end{aligned}
$$

Values for $U_{s}, U_{t}, \lambda_{t}>0, \alpha_{t}$ are found to solve

$$
\min \sum\left(\alpha_{t}\right)^{2}
$$

subject to :

$$
\begin{aligned}
U_{s} & \leq U_{t}+\lambda_{t} \mathbf{p}_{t}^{\prime}\left(\mathbf{x}_{0}-\mathbf{x}_{t}\right)-\alpha_{t}\left(x_{s}^{0}-x_{t}^{0}\right) \\
\alpha_{t} & \leq \lambda_{t} p_{t}^{0}
\end{aligned}
$$




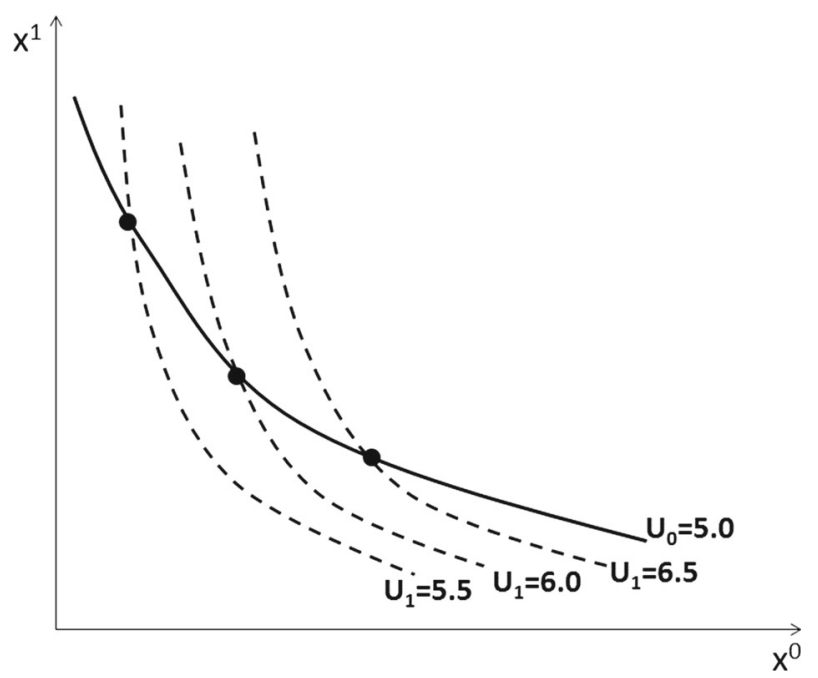

Fig. 1 Preferences shift as environmental quality changes

All the models mentioned above applied to revealed preference work in a similar way, differing in exactly how the adjustment in the utility function is made. The idea is as follows. Figure 1 illustrates three points in a 2-good world on a base-period (period 0) indifference curve shown using a solid curve, with a utility level of, say, 5 . We reiterate as a reminder that superscripts denote the good and subscripts denote the observation period. If $q$ and hence $\alpha$ (for the ABBC model) increases from period 0 to period 1 then the utility of those points increases by $\alpha_{1} x^{0}$ (we normalise $\alpha_{0}$ to 0 ) and the marginal rate of substitution between good 0 and good 1 becomes higher at each point. The new indifference curves for period 1 preferences are shown by the dashed curves. If we knew what the quality adjustment was, and wanted to re-draw everything in terms of period 0 preference space, then this would imply something like that illustrated in Fig. 2.

If we observe a violation of revealed preference theory as illustrated in Fig. 3 where bundle $x_{0}$ is bought in period 0 when bundle $x_{1}$ is affordable and then bundle $x_{1}$ is bought in period 1 when bundle $x_{0}$ is affordable then we can "explain" this by the assumption that the preference map has changed between periods 0 and 1 . Thus $x_{0}$ was chosen with preferences something like the solid indifference curve in Fig. 4, and $x_{1}$ was chosen when the map had shifted to the dashed indifference curve.

So we can ask what the minimum adjustment is that we need to make to preferences in order to justify the violation. In the ABBC model, this process works in the way shown in Figs. 5 and 6. Looking at the violation in Fig. 5, we know that when $x_{0}$ was chosen under initial preferences it was the case that:

$$
\frac{\partial U\left(x_{0}^{0}, x_{0}^{1}\right) / \partial x_{0}^{0}}{\partial U\left(x_{0}^{0}, x_{0}^{1}\right) / \partial x_{0}^{1}}=\frac{p_{0}^{0}}{p_{0}^{1}}
$$

Now when $q_{0}$ changes to $q_{1}$ our hypothesis is that the marginal rate of substitution between $x^{0}$ and $x^{1}$ at $x_{0}$ (i.e. the slope of the period 1 indifference curve through $x_{0}$ ) changes to

$$
\frac{\partial U\left(x_{0}^{0}, x_{0}^{1}\right) / \partial x_{0}^{0}+\alpha_{1}}{\partial U\left(x_{0}^{0}, x_{0}^{1}\right) / \partial x_{0}^{1}}=\frac{p_{0}^{0}}{p_{0}^{1}}
$$




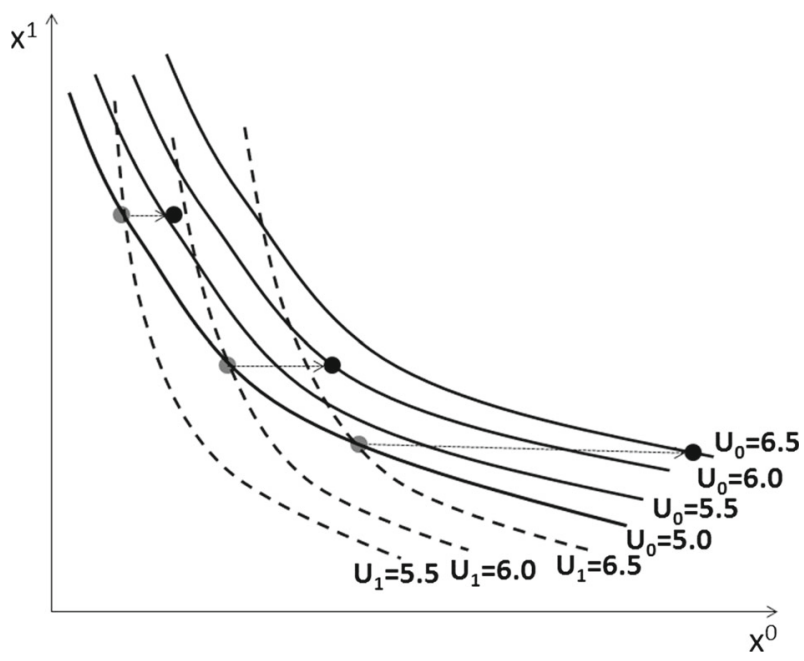

Fig. 2 Redrawing indifference curves in base period quality space

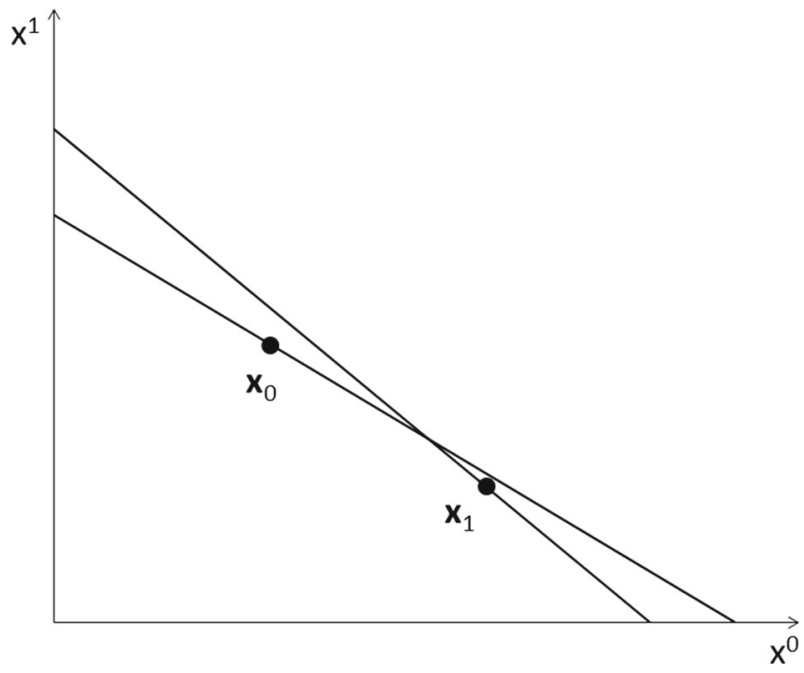

Fig. 3 A revealed preference violation

With the example we have, it can be seen from Fig. 5 that to rationalise the choice of $x_{1}$ given the choice of $x_{0}$, this period 1 indifference curve through $x_{0}$ must be steeper (in absolute value) than the line illustrated that joins $x_{0}$ and $x_{1}$. In other words, with these two data points the lower bound for $\alpha_{1}$ comes from making $x_{0}$ indifferent to $x_{1}$ with period 1 preferences. In this simple two observation example, then, the Afriat inequality from period 0 preferences $U_{1} \leq U_{0}+\lambda_{0} \mathbf{p}_{0}^{\prime}\left(\mathbf{x}_{1}-\mathbf{x}_{0}\right)$ and the bound condition for preference change $U_{0}+\alpha_{1} x_{0}^{0}=U_{1}+\alpha_{1} x_{1}^{0}$ gives

$$
\frac{\alpha_{1}}{\lambda_{0}} \geq \frac{\mathbf{p}_{0}^{\prime}\left(\mathbf{x}_{1}-\mathbf{x}_{0}\right)}{x_{0}^{0}-x_{1}^{0}} .
$$




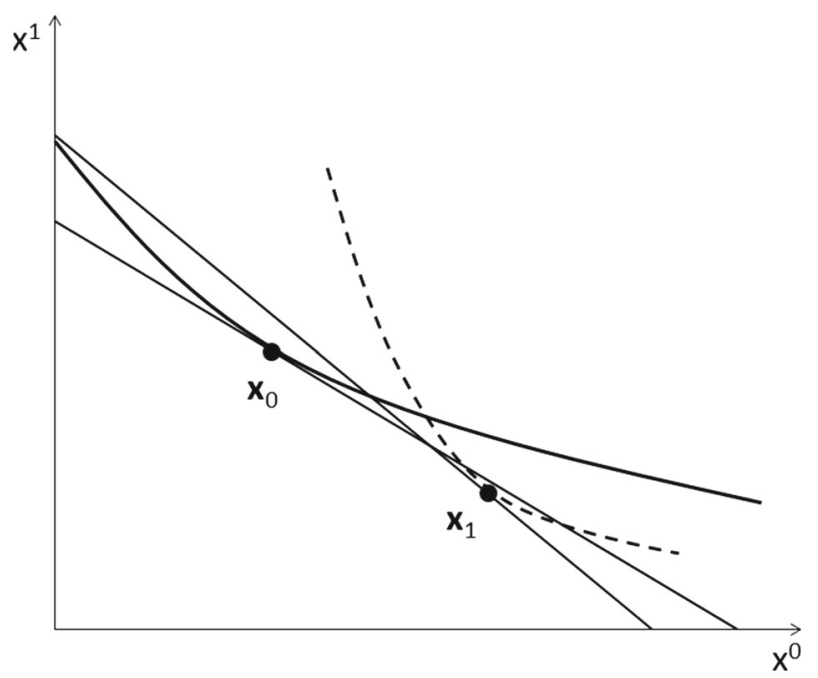

Fig. 4 Explaining a revealed preference violation with a preference shift

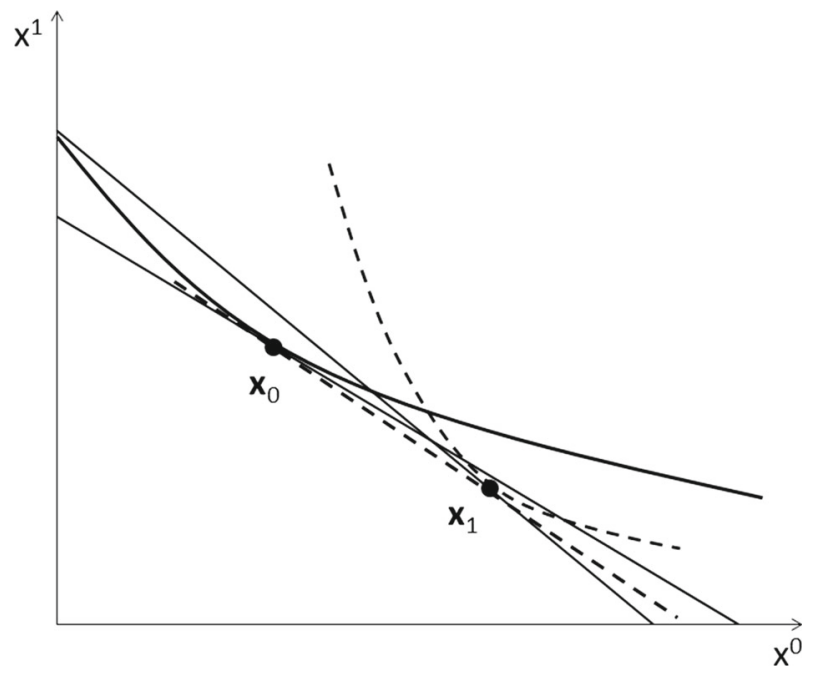

Fig. 5 Bounding the quality change that rationalises a revealed preference violation-intuition

Looking at Fig. 5, it is apparent that if we were able to move the budget line for period 1 slightly inwards (and all goods are weakly normal) then the new choice of $x_{1}$ would require a steeper hypothetical period 1 indifference curve through $x_{0}$ to explain the observed revealed preference violation. This would continue until we pass the period 1 budget line through $\mathbf{x}_{0}$ as illustrated in Fig. $6 .^{5}$ Denoting choices along budget expansion paths by $\mathbf{x}_{t}(y)$ then, taking $\mathbf{x}_{0}$ as the fixed point for comparison, the highest lower bound on $\alpha_{1}$ would be given by

5 In the two good case, this obviously means that the lower bound for the indifference curve lies on the period 1 budget line. 


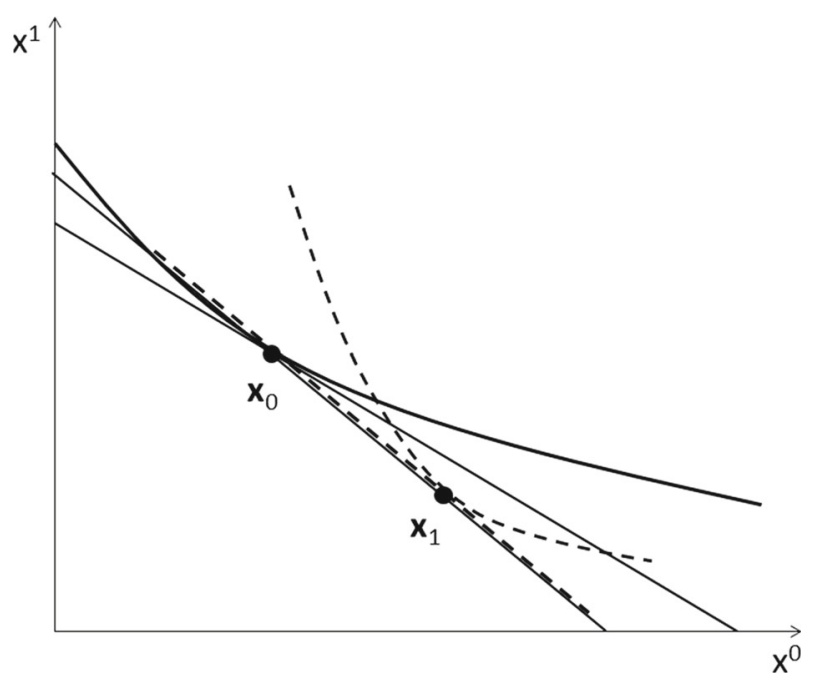

Fig. 6 Bounding the quality change that rationalises a revealed preference violation — the highest lower bound

$$
\frac{\alpha_{1}}{\lambda_{0}} \geq \frac{\mathbf{p}_{0}^{\prime}\left(\mathbf{x}_{1}\left(\mathbf{p}_{1}^{\prime} \mathbf{x}_{0}\right)-\mathbf{x}_{0}\right)}{x_{0}^{0}-x_{1}^{0}\left(\mathbf{p}_{1}^{\prime} \mathbf{x}_{0}\right)}
$$

We can put all these types of adjustment model into the general framework proposed by Erwin Diewert (1973). Diewert wrote augmented Afriat inequalities in the following form: find $U_{s}, U_{t}, \lambda_{t}>0, S_{t s}^{-}>0, S_{t s}^{+}>0$

$$
\min \sum_{t=0}^{T} \sum_{s \neq t, s=1}^{T} S_{t s}^{-}
$$

subject to :

$$
U_{s} \leq U_{t}+\lambda_{t} \mathbf{p}_{t}^{\prime}\left(\mathbf{x}_{s}-\mathbf{x}_{t}\right)+S_{t s}^{-}-S_{t s}^{+}
$$

The variables $S_{t s}^{-}$and $S_{t s}^{+}$are slack variables. The minimum that $\sum_{t=0}^{T} \sum_{s \neq t, s=1}^{T} S_{t s}^{-}$can be is zero, and if this minimum can be achieved then there is a solution to the normal Afriat inequalities:

$$
U_{s} \leq U_{t}+\lambda_{t} \mathbf{p}_{t}^{\prime}\left(\mathbf{x}_{s}-\mathbf{x}_{t}\right)
$$

If there is no solution to the Afriat inequalities, then the $S_{t s}^{-}$are a measure of the deviations from a possible solution. If, instead, we give this slack variable a particular form then we would get a particular model, for example one of the models discussed above, or, if we set $S_{t s}^{-}=\lambda_{t} \mathbf{p}_{t}^{\prime} \mathbf{x}_{t}(1-e)$ to give

$\min e$

subject to :

$$
U_{s} \leq U_{t}+\lambda_{t} \mathbf{p}_{t}^{\prime}\left(\mathbf{x}_{s}-\mathbf{x}_{t}\right)+\lambda_{t} \mathbf{p}_{t}^{\prime} \mathbf{x}_{t}(1-e)
$$

then this would estimate the Afriat efficiency index, $e$, which measures what proportion of his budget the consumer would have to "waste" in order to satisfy GARP. There are many 
different adjustments that can be made to the data in order to correct revealed preference rejections. Clearly the ABBC model makes sense in the context of a hedonic price model, and since the extra unknowns, $\alpha_{t}$, enter the Afriat equations in a linear fashion, it is particularly easy to work with.

It is also clear that Eq. (3) could be amended to include a translating element $\beta_{t}$, becoming

$$
U\left(x^{0}, \mathbf{x}^{K}, q_{t}\right)=U\left(x_{t}^{0}+\beta_{t}, \mathbf{x}_{t}^{K}\right)+\alpha_{t} x_{t}^{0}
$$

In this case the Afriat-type conditions become

$$
U_{s} \leq U_{t}+\lambda_{t} \mathbf{p}_{t}^{\prime}\left(\mathbf{x}_{s}-\mathbf{x}_{t}\right)-\alpha_{t}\left(x_{s}^{0}-x_{t}^{0}\right)+\lambda_{t} p_{t}^{0}\left(\beta_{s}-\beta_{t}\right)
$$

and the researcher could minimise $\sum\left(\alpha_{t}\right)^{2}+\sum\left(\beta_{t}\right)^{2}$ subject to equation (7). Translating allows for some indirect use value of $q$ even when $x^{0}=0$. It is important to remember though that, as highlighted by the slack variable approach, there are always a set of adjustments that will correct revealed preference rejections and so any particular set rest on the particular assumptions and interpretations made.

In Sect. 6, we discuss how to implement empirically the marginal utility perturbation model. Since the recovered marginal utility perturbations can be used to adjust the price of $x_{t}^{0}$ to its shadow value $\left(p_{t}^{0}-\alpha_{t} / \lambda_{t}\right)$, these adjusted prices form the basis for a welfare analysis. In the next section we therefor describe how revealed preference methods can be used to bound measures of welfare costs such as compensating variation.

\section{Bounding the Compensating Variation}

To introduce the method we start with the standard case where we would like to compute the compensating variation of some price change from $\mathbf{p}_{0}$ to $\mathbf{p}_{N}$ where $\mathbf{p}_{N}$ may or may not be in the observed set $\left\{\mathbf{x}_{t} ; \mathbf{p}_{t}\right\}_{t=0, \ldots, T}$

$$
C V_{0, N}=c\left(u_{0}, \mathbf{p}_{N}\right)-c\left(u_{0}, \mathbf{p}_{0}\right)
$$

What we need to do is bound the indifference curve through $\mathbf{x}_{0}$. To do this using the revealed preference approach we want to know if there are any bundles $\mathbf{x}$ for which we can definitely say that $\mathbf{x} R \mathbf{x}_{0}$ and if there are any bundles for which we can definitely say that $\mathbf{x}_{0} R \mathbf{x}$. We call the first set the revealed preferred (to $\left.\mathbf{x}_{0}\right)$ set $R P\left(\mathbf{x}_{0}\right)$, and the second the revealed worse (to $\mathbf{x}_{0}$ ) set $R W\left(\mathbf{x}_{0}\right)$.

Following the approach in Varian (1982): given any bundle $\mathbf{x}_{0}$, we define the set of prices $\mathbf{p}_{s 0}$ that support $\mathbf{x}_{0}$ (clearly this includes $\mathbf{p}_{0}$ ) by:

$$
S^{p}\left(\mathbf{x}_{0}\right)=\left\{\mathbf{p}_{s 0}: \begin{array}{l}
\mathbf{p}_{s 0}^{\prime} \mathbf{q}_{0}=x_{0} \\
\left\{\mathbf{p}_{s 0}, \mathbf{p}_{t} ; \mathbf{q}_{0}, \mathbf{q}_{t}\right\}_{t=0, \ldots, T} \text { satisfies GARP }
\end{array}\right\}
$$

This is simply the set of prices at which $\mathbf{x}_{0}$ could be demanded and still be consistent with the previously observed behaviour. Constructing this set is a linear programming problem. 


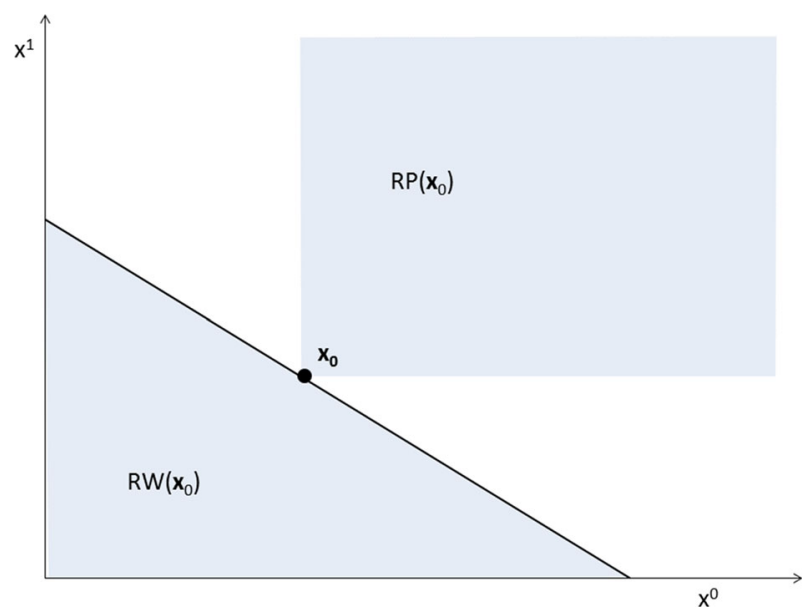

Fig. 7 Bounding an indifference curve a single observation

Then the revealed worse and revealed preferred sets can be constructed as follows:

$$
\begin{gathered}
R W\left(\mathbf{x}_{0}\right)=\left\{\begin{array}{c}
\mathbf{x} \mid \forall \mathbf{p}_{s 0} \in S^{p}\left(\mathbf{x}_{0}\right), \\
\mathbf{p}_{s 0}^{\prime} \mathbf{x}_{0} \geq \mathbf{p}_{s 0}^{\prime} \mathbf{x}_{t} \text { for some } \mathbf{x}_{t} P \mathbf{x} \\
\text { or } \mathbf{p}_{s 0}^{\prime} \mathbf{x}_{0}>\mathbf{p}_{s 0}^{\prime} \mathbf{x}_{t} \text { for some } \mathbf{x}_{t} R \mathbf{x}
\end{array}\right\} \\
R P\left(\mathbf{x}_{0}\right)=\left\{\begin{array}{c}
\mathbf{x} \mid \forall \mathbf{p} \in S^{p}(\mathbf{x}), \\
\mathbf{p}^{\prime} \mathbf{x} \geq \mathbf{p}_{t}^{\prime} \mathbf{x} \text { for some } \mathbf{x}_{t} P \mathbf{x}_{0} \\
\text { or } \mathbf{p}^{\prime} \mathbf{x}>\mathbf{p}_{t}^{\prime} \mathbf{x} \text { for some } \mathbf{x}_{t} R \mathbf{x}_{0}
\end{array}\right\}
\end{gathered}
$$

Figure 7 shows the bounds on $R P\left(\mathbf{x}_{0}\right)$ and $R W\left(\mathbf{x}_{0}\right)$ where we only observe $\mathbf{x}_{0}$. For the revealed worse set, we know the consumer has revealed that he prefers his choice to anything else in his budget set. For the revealed preferred set we can only use non-satiation to give as the bound shown. Figure 6 shows three more observed choices $\left\{\mathbf{x}_{1}, \mathbf{x}_{2}, \mathbf{x}_{3}\right\}$ Using the approach above, these allow us to tighten the bounds as shown. For these data, we see that $\mathbf{x}_{3} P^{0} \mathbf{x}_{1} P^{0} \mathbf{x}_{0}$. Thus $R P\left(\mathbf{x}_{0}\right)$ now becomes the convex hull of these points, plus the nonsatiation condition. We also see that $\mathbf{x}_{0} P^{0} \mathbf{x}_{2}$. Since $\mathbf{x}_{2}$ is at least as good as everything in the period 2 budget set, we can add the part of this budget set that is not a subset of the period 0 budget set to $R W\left(\mathbf{x}_{0}\right)$. The problem often faced in actual consumer data is that we do not observe choices that are serendipitously arranged to give us the kind of information shown in Fig. 8. Data are sometimes arranged in a way that gives us little extra information as to the shape of the $R P\left(\mathbf{x}_{0}\right)$ and $R W\left(\mathbf{x}_{0}\right)$ sets and hence the indifference curve through $\mathbf{x}_{0}$.

A method for dealing with this problem was proposed and refined in Blundell et al. (2003, 2008) and Blundell et al. (2015). The idea in these papers is to use some nonparametric estimate of budget expansion paths so that we can estimate $\mathbf{x}_{t}(y)$, the demands at prices $\mathbf{p}_{t}$ (we omit price as an argument to simplify notation), at any level of budget $y$. As shown in Blundell et al. (2003), this will allow us to improve the bounds on $R W\left(\mathbf{x}_{0}\right)$ and $R P\left(\mathbf{x}_{0}\right)$ (Blundell et al. 2015 show how bounds can be further tightened in the case of estimating $\mathrm{EV}$ for a new, previously unobserved bundle, but we with stick with the CV example for ease of exposition). Intuitively, the idea is to move budget lines closer in to the comparison bundle $\mathbf{x}_{0}$. To start, each $y_{t}$ is set so that $\mathbf{p}_{t}^{\prime} \mathbf{x}_{t}\left(\widehat{y}_{t}^{P}\right)=\mathbf{p}_{t}^{\prime} \mathbf{x}_{0}$, i.e. $\mathbf{x}_{t}\left(\widehat{y}_{t}^{P}\right) R^{0} \mathbf{x}_{0}$. Then, if, for 


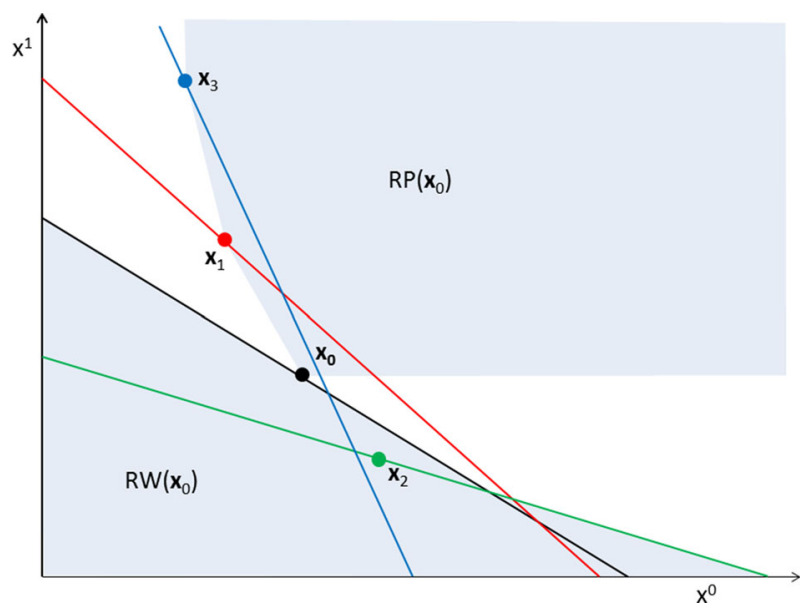

Fig. 8 Bounding an indifference curve more information

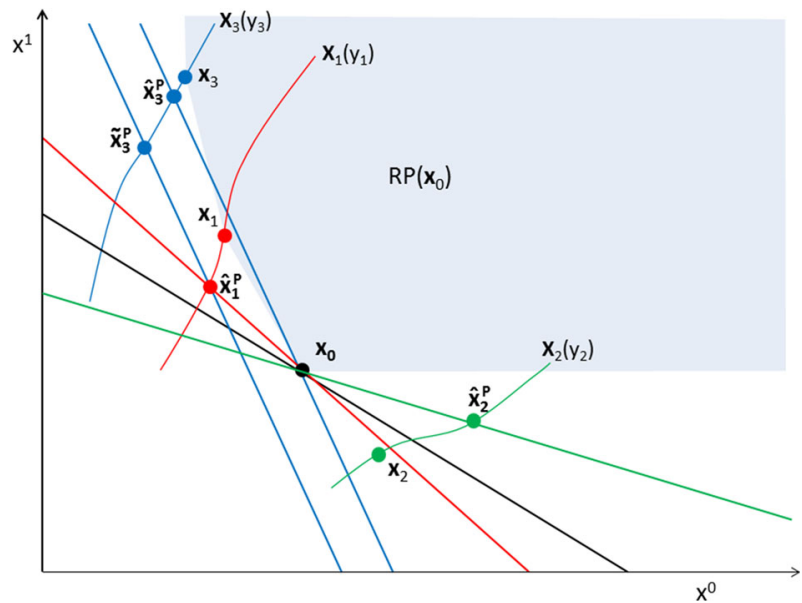

Fig. 9 Bounding an indifference curve improving the raw upper bound: initial improvement

this set we find any $\mathbf{x}_{t}\left(\widehat{y}_{t}^{P}\right) P^{0} \mathbf{x}_{s}\left(\widehat{y}_{s}^{P}\right)$, then $\widehat{y}_{t}^{P}$ is reduced until $\mathbf{p}_{t}^{\prime} \mathbf{x}_{t}\left(\widetilde{y}_{t}^{P}\right)=\mathbf{p}_{t}^{\prime} \mathbf{x}_{s}\left(\widehat{y}_{s}^{P}\right)$ (or the minimum of these if there are multiple $\mathbf{x}_{s}\left(\widehat{y}_{s}^{P}\right)$ to which $\mathbf{x}_{t}\left(\widehat{y}_{t}^{P}\right)$ is revealed strictly preferred). When there are no more iterations to be made, call this set $X P\left(\mathbf{x}_{0}\right)$. The convex hull of this set gives the tightest bound on $R P\left(\mathbf{x}_{0}\right)$ for this dataset.

This approach is illustrated in Fig. 9. We show the placement of bundles on their budget lines through $\mathbf{x}_{0}$ for the initial iteration by $\left\{\widehat{\mathbf{x}}_{1}^{P}, \widehat{\mathbf{x}}_{2}^{P}, \widehat{\mathbf{x}}_{3}^{P}\right\}$, i.e. $\mathbf{p}_{t}^{\prime} \widehat{\mathbf{x}}_{t}^{P}=\mathbf{p}_{t}^{\prime} \mathbf{x}_{0}$. Now note that $\widehat{\mathbf{x}}_{3}^{P} P^{0} \widehat{\mathbf{x}}_{1}^{P}$ so that in the next iteration we move $\widehat{y}_{3}^{P}$ to $\tilde{y}_{3}^{P}=\mathbf{p}_{3}^{\prime} \widehat{\mathbf{x}}_{1}^{P}$ which results in a demand of $\widetilde{\mathbf{x}}_{3}^{P}$. In this example, there are now further improvements that can be made. Figure 10 shows the updated, improved $R P\left(\mathbf{x}_{0}\right)$ set.

A similar procedure applies for $R W\left(\mathbf{x}_{0}\right)$. In this case we set $y_{t}$ so that $\mathbf{p}_{0}^{\prime} \mathbf{x}_{t}\left(\widehat{y}_{t}^{W}\right)=\mathbf{p}_{0}^{\prime} \mathbf{x}_{0}$, i.e. $\mathbf{x}_{0} R^{0} \mathbf{x}_{t}\left(\widehat{y}_{t}^{W}\right)$. Then, if, for this set any $\mathbf{x}_{t}\left(\widehat{y}_{t}^{W}\right) P^{0} \mathbf{x}_{s}\left(\widehat{y}_{s}^{W}\right)$, then $\widehat{y}_{s}^{W}$ is increased until $\mathbf{p}_{t}^{\prime} \mathbf{x}_{t}\left(\tilde{y}_{t}^{W}\right)=\mathbf{p}_{t}^{\prime} \mathbf{x}_{s}\left(\widehat{y}_{s}^{W}\right)$ (or the maximum of these if there are multiple $\mathbf{x}_{s}\left(\widehat{y}_{s}^{W}\right)$ ). When all iterations are complete, call this set $X W\left(\mathbf{x}_{0}\right)$. The (closure of) the complement of the convex 


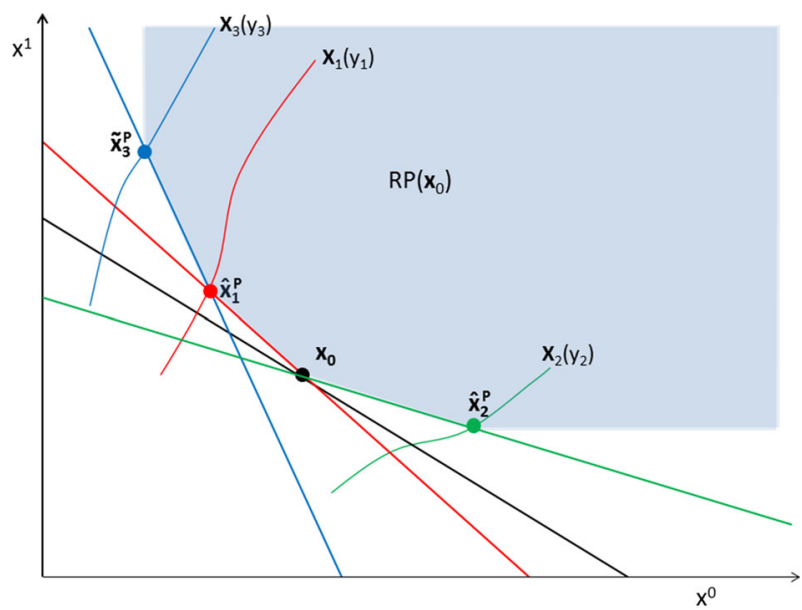

Fig. 10 Bounding an indifference curve improving the raw upper bound: final improvement

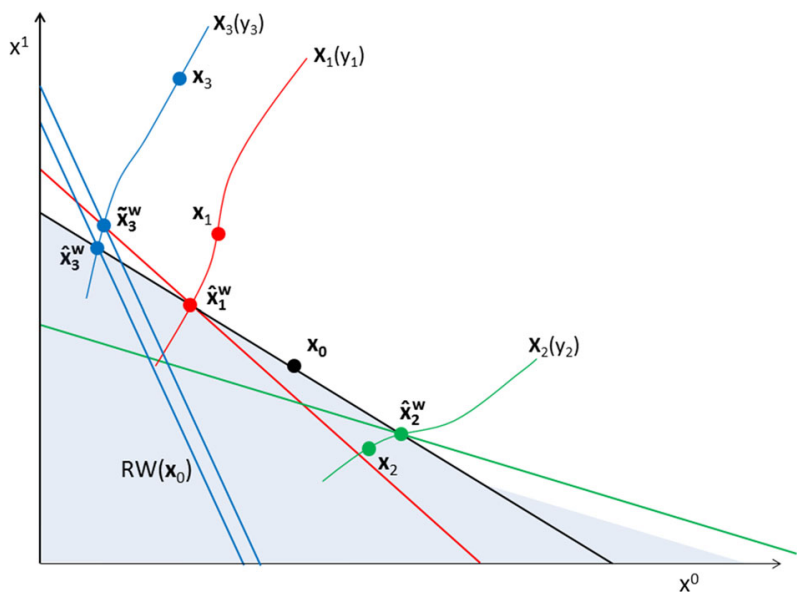

Fig. 11 Bounding an indifference curve improving the raw lower bound: initial improvement

hull of these points is then the tightest bound on $R W\left(\mathbf{x}_{0}\right) \cdot{ }^{6}$ Figure 11 shows this process for our example data, where $\left\{\widehat{\mathbf{x}}_{1}^{W}, \widehat{\mathbf{x}}_{2}^{W}, \widehat{\mathbf{x}}_{3}^{W}\right\}$ shows the initial placement at $\mathbf{p}_{0}^{\prime} \widehat{\mathbf{x}}_{t}^{W}=\mathbf{p}_{0}^{\prime} \mathbf{x}_{0}$. This time we find that $\widehat{\mathbf{x}}_{1}^{W} P^{0} \widehat{\mathbf{x}}_{3}^{W}$ and so we move $\widehat{y}_{3}^{W}$ to $\widetilde{y}_{3}^{W}=\mathbf{p}_{3}^{\prime} \widehat{\mathbf{x}}_{1}^{P}$ which results in a demand of $\widetilde{\mathbf{x}}_{3}^{W}$. Figure 12 shows the updated, improved $R W\left(\mathbf{x}_{0}\right)$ set.

The combined final $R P\left(\mathbf{x}_{0}\right)$ and $R W\left(\mathbf{x}_{0}\right)$ sets are shown together in Fig. 13. For some price vector $\mathbf{p}_{N}$, the upper bound on $c\left(u_{0}, \mathbf{p}_{N}\right)$ is therefore $\min \mathbf{p}_{N}^{\prime} \mathbf{x}_{t}$ such that $\mathbf{x}_{t} \in X P\left(\mathbf{x}_{0}\right)$. Similarly the lower bound is $\min \mathbf{p}_{N}^{\prime} \mathbf{x}_{t}$ such that $\mathbf{x}_{t} \in X W\left(\mathbf{x}_{0}\right)$. This is illustrated in Fig. 14.

Extending this method to bounding welfare measures of environmental quality change where we model the quality change as a price adjustment to a related marketed good is intuitively relatively straightforward. Suppose we take the ABBC model and we have used the nonparametric and revealed preference methods described in the next section to estimate

6 with some extra steps to identify the final points on each boundaries where only one $x_{0}^{k}$ is positive. 


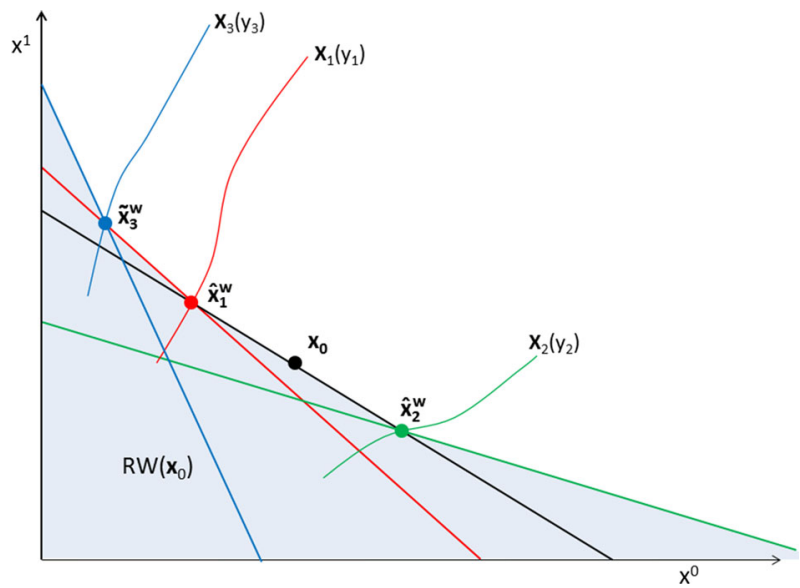

Fig. 12 Bounding an indifference curve improving the lower upper bound: final improvement

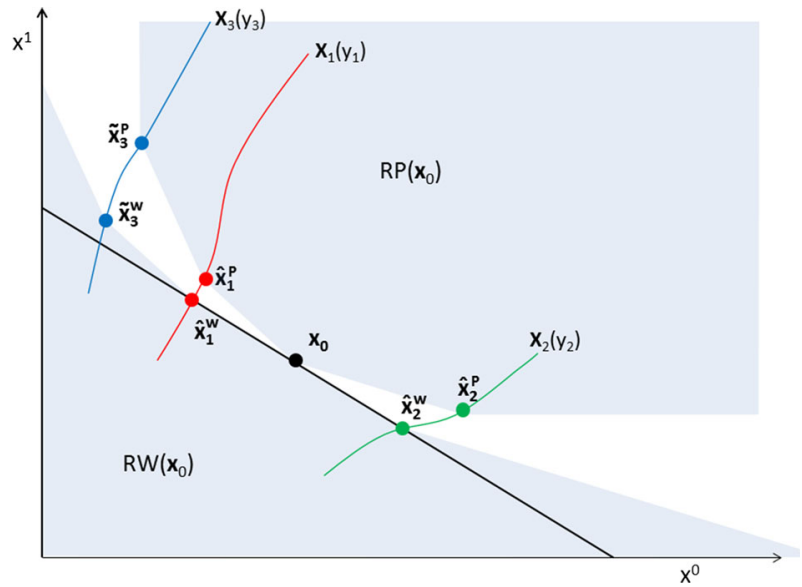

Fig. 13 Combined tightest bounds on an indifference curve

$\alpha_{t} / \lambda_{t}$, or, more specifically $\alpha_{t} / \lambda_{t}$ conditional on the quantile of budget share for good $x_{t}^{0}$ for some population of interest. The ABBC model gives a cost function

$$
\begin{array}{r}
c\left(u, p_{t}^{0}, \mathbf{p}_{t}^{K}, q_{t}\right)=\min \mathbf{p}_{t}^{\prime} \mathbf{x}_{t} \text { s.t. } u=U\left(x_{t}^{0}, \mathbf{x}_{t}^{K}, q_{0}\right)+\alpha_{t} x_{t}^{0} \\
\Rightarrow c\left(u, p_{t}^{0}, \mathbf{p}_{t}^{K}, q_{t}\right)=c\left(u, p_{t}^{0}-\frac{\alpha_{t}}{\lambda_{t}}, \mathbf{p}_{t}^{K}, q_{0}\right)
\end{array}
$$

and likewise

$$
x_{t}^{k}\left(y_{t}, p_{t}^{0}, \mathbf{p}_{t}^{K}, q_{t}\right)=x_{t}^{k}\left(y_{t}, p_{t}^{0}-\frac{\alpha_{t}}{\lambda_{t}}, \mathbf{p}_{t}^{K}, q_{0}\right)
$$

Thus the method for the standard case outlined above will apply, replacing $p_{t}^{0}$ with $p_{t}^{0}-$ $\alpha_{t} / \lambda_{t}$. And, as explained in the next section, the very method for calculating $\alpha_{t} / \lambda_{t}$ means we will already have generated the nonparametric expansion paths we need for finding the best bounds on the compensating variation. 


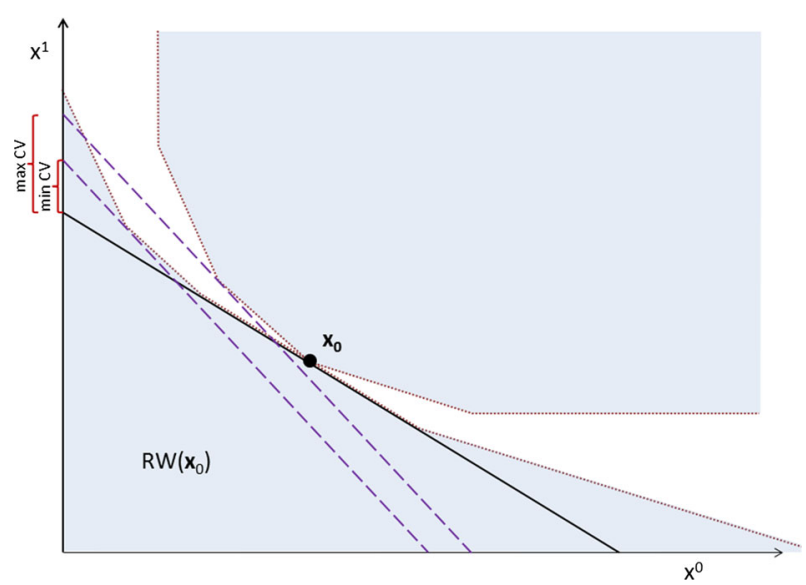

Fig. 14 Using indifference curve bounds to bound compensating variation

We illustrate the process for the basic two period example we depicted in Fig. 6. Figure 6 illustrated the minimal change to marginal utility that is necessary to explain the apparent violation of revealed preference. This means that if quality if period 0 was $q_{1}$ instead of $q_{0}$ this can be represented as $p_{0}^{0}$ changing to $p_{0}^{0}-\alpha_{1} / \lambda_{0}$. Using Eq. (5) in the two good case gives

$$
\begin{aligned}
\widetilde{p}_{0}^{0} & =p_{0}^{0}-\frac{p_{0}^{0}\left(x_{1}^{0}-x_{0}^{0}\right)+p_{0}^{1}\left(x_{1}^{1}-x_{0}^{1}\right)}{\left(x_{0}^{0}-x_{1}^{0}\right)} \\
& =2 p_{0}^{0}-\frac{p_{0}^{1}\left(x_{1}^{1}-x_{0}^{1}\right)}{\left(x_{0}^{0}-x_{1}^{0}\right)}
\end{aligned}
$$

but since $\left(x_{1}^{1}-x_{0}^{1}\right) /\left(x_{0}^{0}-x_{1}^{0}\right)=p_{1}^{0} / p_{1}^{1}$ this gives

$$
\begin{aligned}
& \widetilde{p}_{0}^{0}=2 p_{0}^{0}-\frac{p_{0}^{1} p_{1}^{0}}{p_{1}^{1}} \Rightarrow \\
& \frac{\widetilde{p}_{0}^{0}}{p_{1}^{0}}=2 \frac{p_{0}^{0}}{p_{1}^{0}}-\frac{p_{0}^{1}}{p_{1}^{1}}<\frac{p_{0}^{0}}{p_{1}^{0}} \text { since } \frac{p_{0}^{0}}{p_{1}^{0}}<\frac{p_{0}^{1}}{p_{1}^{1}} .
\end{aligned}
$$

The relative price of good 0 has declined since we are illustrating a quality improvement.

As we only have one price regime for $q_{0}$, the bounds on the compensating variation of the quality change would be the simple bounds given by the extremes of perfect complementarity and perfect substitutability between $x^{0}$ and $x^{1}$. This is illustrated in Fig. 15.

\section{Empirical Approach}

Here we outline our empirical approach to bounding the value of unobserved environmental quality. Following the discussion so far, there are three key steps: First, we develop the empirical approach for recovering the individual changes in the marginal utility $\alpha_{t}$ as in (3) above. We allow these changes to be individual and time specific so as not to restrict individual marginal utility valuations. Second, we construct quality adjusted prices $p_{t}^{0}-\frac{\alpha_{t}}{\lambda_{t}}$ 


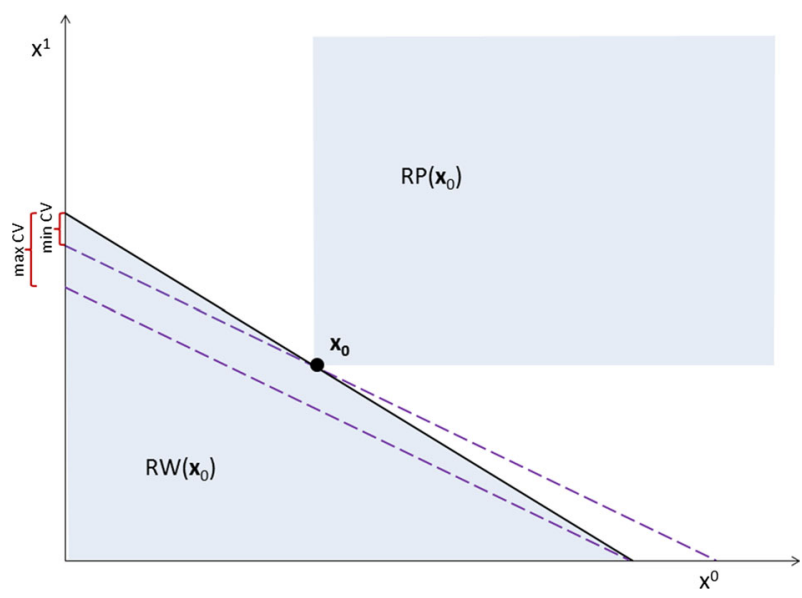

Fig. 15 Bounding the compensating variation of a quality change in a simple example

for the complementary good $x_{t}^{0}$ that are just sufficient to rationalise observed choices of the marketed goods $x_{t}^{0}, \mathbf{x}_{t}^{K}$. Finally, given the adjusted prices, we estimate the bounds on individual compensating variations as described in Sect. 5 above.

\subsection{Nonparametric Estimation of the Quality Perturbations to Marginal Utility}

We can apply the ideas developed in this paper to panel data or to repeated cross-section surveys on household consumer expenditure survey data. We assume we observe each consumer choice behaviour for the set of market goods $\mathbf{x}$ with relative prices $\mathbf{p}$, denoted $D=\left\{\mathbf{p}_{t}, \mathbf{x}_{t}\right\}_{t=1, \ldots, T}$, where $T$ is the number of different relative prices. The $\alpha_{t}$ values can be interpreted as the marginal utility perturbation to good $-0, x^{0}$, relative to that dictated by base utility at observed demands

$$
U\left(x^{0}, \mathbf{x}^{K}, q_{t}\right)=U\left(x_{t}^{0}, \mathbf{x}_{t}^{K}\right)+\alpha_{t} x_{t}^{0}
$$

Suppose we have repeated cross-section data on the same sub-population of individuals who consume $x^{0}, \mathbf{x}^{K}$ and face environmental quality $q_{t}$ in each price regime period $t$. We use quantile demands to recover individual demand behaviour at each price regime. We then apply the revealed preference methodology to estimated quantities in order to make statements about quality change over time for the population of interest.

We begin with the case where there are just two marketed goods of interest. $x^{0}$ that is related directly to environmental quality and $x^{1}$. We assume that individual demands are monotonic in scalar unobserved heterogeneity. To allow for heterogeneity, we augment individual preferences in to take the form:

$$
u\left(\mathbf{x} ; \alpha_{t}\right)=f\left(x^{1}, x^{0}, \tau\right)+\alpha_{t} x^{0}
$$

where $\tau \sim U(0,1)$ represents time-invariant interpersonal taste heterogeneity and $\alpha_{t}$ gives the perturbation to the marginal utility of good 0 experienced by that individual consumer at time $t$, as the quality of the environmental quality.

Suppose we have $t=1, \ldots, T$ cross-section observations on individuals $i=1, \ldots, N_{t}^{c}$. For quantile demands to identify individual demands we have to place further restrictions 
on preferences that guarantee demands are monotonic in unobserved scalar heterogeneity. Matzkin (2007) provides the following general conditions on heterogeneous preferences $f\left(x^{1}, x^{0}, \tau\right)$ to be invertible in unobserved heterogeneity

$$
f\left(x^{1}, x^{0}, \tau\right)=f\left(x^{1}, x^{0}\right)+w\left(x^{0}, \tau\right)
$$

where the functions $f$ and $w$ are twice continuously differentiable, strictly increasing and strictly concave, and $w\left(x^{0}, \tau\right)$ has a strictly positive second derivative. Under these conditions, the demand function for $x^{0}$ will satisfy the restrictions of consumer choice for each value $\tau$. Similarly, demands (and budget shares) will be monotonic in $\tau$.

To equate quantile and individual demands we further assume that the quality evolution term $\left(\tau+\alpha_{t}\right) x^{0}$ does not alter the ranking of consumers over time in the $x^{0}$ distribution conditional on (p,y). With these assumptions, and drawing on Blundell et al. (2014), the $\tau$ 'th conditional quantile reflects the demand behaviour of an individual with unobserved, time-invariant preference heterogeneity $\tau$ and $\alpha_{t} x^{0}$.

For each of the $t=1, \ldots, T$ price regimes, we write quantile expansion paths (Engel curves) for $x^{0}$ as

$$
x^{0}=Q_{x^{0}}(\tau \mid y)
$$

where

$$
\tau \sim U(0,1) \mid y
$$

and $y$ is total expenditure on $x^{0}$ and $x^{1}$. As in ABBC we may include a vector of household characteristics.

To allow for the possible endogeneity of total expenditure, $y$, we follow Blundell and Powell (2007) and Imbens and Newey (2009) and use a quantile control function approach. For example, we may use disposable income as an excluded instrument that allows us to recover the control variable.

To allow for a reasonably flexible specification of each quantile expansion path, we could, as an illustration, choose a second order polynomial and write $\mathbf{y}=\left[\log (y), \log (y)^{2}\right]$. Then, for each subsample of consumers at each price regime, $\boldsymbol{\beta}(\tau)$ is estimated as:

$$
\boldsymbol{\beta}(\tau)=\underset{\boldsymbol{\beta}}{\arg \min } \sum_{i} \rho_{\tau}\left(x_{i}^{0}-\mathbf{y}_{i}^{\prime} \boldsymbol{\beta}\right)
$$

where $\rho_{\tau}$ is the standard asymmetric absolute loss function for quantile regression of Koenker and Bassett (1978).

Reviving the time subscripts, the set of estimated quantities, at which taste changes are recovered, are constructed as:

$$
x_{t}^{0}(\tau)=\widetilde{\mathbf{y}}_{t}^{\prime} \widehat{\boldsymbol{\beta}}_{t}(\tau)
$$

for $t=1, \ldots, T$ and where $\tilde{\mathbf{y}}_{t}=\left[\log \left(\tilde{y}_{t}\right), \log \left(\tilde{y}_{t}\right)^{2}\right]$. Note that the $\tilde{y}_{t}$ are expenditure levels on the sequential maximum power (SMP) path set to ensure that the average demand in period $t=1, \ldots, T-1$ is weakly affordable at the time $t$ budget. By using these expenditure levels, rather than, for example, mean expenditure in a given period, we ensure that budget lines at different time periods intersect. This is a necessary condition for detecting violations of a time-invariant utility function in the data. For more details see Blundell et al. (2003, 2008).

The quadratic programming procedure of Sect. 4.2 can then be applied to the set $\left\{\mathbf{p}_{t}, \mathbf{x}_{t}^{\tau}\right\}_{t=1, \ldots, T}$, with $\mathbf{x}_{t}^{\tau}$ estimated as above. The minimal squared perturbations to the 
marginal utility of good-0 relative to preferences in period 1 that are necessary to good0 rationalise observed choice behaviour $D=\left\{\mathbf{p}_{t}, \mathbf{x}_{t}\right\}_{t=1, \ldots, T}$ are identified as the unique solution set $\left\{\alpha_{t}\right\}_{t=1, \ldots, T}$ to the following quadratic programme.

$$
\min _{\left\{v_{t}, \lambda_{t}, \alpha_{t}\right\}_{t=1, \ldots, T}} \sum_{t=1}^{T}\left(\alpha_{t}\right)^{2}
$$

subject to:

1. The revealed preference inequalities:

$$
\begin{aligned}
v_{s}-v_{t}+\alpha_{t}\left(x_{s}^{0}-x_{t}^{0}\right) & \leq \lambda_{t} \mathbf{p}_{t}^{\prime}\left(\mathbf{x}_{s}-\mathbf{x}_{t}\right) \\
\alpha_{t} & \leq \lambda_{t} p_{t}^{0}
\end{aligned}
$$

2. The normalisation conditions:

$$
\begin{aligned}
& v_{1}=\delta \quad(\text { an arbitrary constant }) \\
& \lambda_{1}=\beta \quad \text { (a strictly positive constant) } \\
& \alpha_{1}=0
\end{aligned}
$$

for $s, t=1, \ldots, T$.

These estimated $\alpha_{t}^{\prime} s$ will differ for each income level $y$ and each quantile $\tau$.

Minimising the sum of squared $\alpha_{t}$ 's subject to the set of revealed preference inequalities ensures that the recovered pattern of minimal taste perturbations are sufficient to rationalise observed choice behaviour. The normalisation conditions are required because the quadratic programming problem is ill-posed in their absence. This is due to the invariance of tastes to positive monotonic changes in the utility function. Let $\left\{\bar{v}_{t}, \bar{\lambda}_{t}, \bar{\alpha}_{t}\right\}_{t=1, \ldots, T}$ represent some feasible solution to the rationalisation constraints. This procedure can bootstrapped to address the issue of sampling variation in estimated quantity sequences. Note that we can stratify by education group, region, and so on.

\subsection{Estimating Bounds on the Compensating Variation}

For each quantile demand we have seen how to estimate of the marginal utility perturbations that will rationalise be sufficient for observed market demands that is subject to environmental quality change. These are allowed to differ across individuals and at every level of income (total expenditure). Thus we can estimate an individual specific series of adjusted prices $p_{t}^{0}-\frac{\widehat{\alpha_{t}}}{\lambda_{t}}$ to the good $x_{t}^{0}$ associated with environmental quality. We label these $\widehat{p}_{t}^{o}$. Again these will differ by income level and quantile. That is we compute the distribution of marginal utility changes consistent with the underlying quality changes as the impact on different individuals (quantiles) at different points in the income distribution $(y)$.

Under the assumptions outlined earlier in the paper, we can now use this series of adjusted prices to place bounds on the estimated distribution of welfare costs of the change in environmental quality. The cost function for any quality change $q_{t}$ in terms of the adjusted price series $\widehat{p}_{t}^{o}$ is given by

$$
c\left(u, p_{t}^{0}, p_{t}^{1}, q_{t}\right)=c\left(u, p_{t}^{0}-\frac{\widehat{\alpha_{t}}}{\lambda_{t}}, p_{t}^{1}, q_{0}\right)
$$

For any income level $y$ and any quantile $\tau$ we can now compute the compensating variation bounds. These bounds will be derived from the estimated $R P\left(\mathbf{x}_{0}\right)$ and $R W\left(\mathbf{x}_{0}\right)$ sets as in Fig. 11 above. For some adjusted price vector $\widehat{\mathbf{p}}_{N}$, the upper bound on $c\left(u_{0}, \widehat{\mathbf{p}}_{N}\right)$ is 
therefore $\min \mathbf{p}_{N}^{\prime} \mathbf{x}_{t}$ such that $\mathbf{x}_{t} \in X P\left(\mathbf{x}_{0}\right)$. Similarly the lower bound is $\min \mathbf{p}_{N}^{\prime} \mathbf{x}_{t}$ such that $\mathbf{x}_{t} \in X W\left(\mathbf{x}_{0}\right)$.

Finally we note, as mentioned before, that with multiple observations on changes in the indicators of environmental quality, we can relate the estimated price adjustments $\frac{\widehat{\alpha_{t}}}{\lambda_{t}}$ to the indicators, and this can also be done by strata of household type or household characteristics could be included as variables. This would allow an assessment of how well and in what way observable indicators of environmental quality explain the variation in price adjustment we estimate and also aid in transferring benefit estimates to different situations and population mixes.

\section{Conclusions}

This paper has provided a theoretical and empirical framework for characterising environmental quality change using nonparametric revealed preference analysis. We have focused on the case in which the non-market good affects the marginal utility of consuming a related market good, which is framed as a shift in the taste for, or quality of, the market good. A quantile approach is adopted to allow for unobserved heterogeneity.

The approach enables the recovery of the minimal variation in quality required to rationalise observed choices of related market goods. The variation in quality appears as a adjustment to the price for related market goods which then allows a revealed preference approach to bounding compensation measures of welfare effects to be applied.

The empirical approach follows three key steps. First the individual changes in the marginal utility are estimated in away that allows them to be individual and time specific. Second we use these estimated marginal utility perturbations to construct quality adjusted prices for the complementary good. We have shown that these are just sufficient to rationalise observed choices of the marketed goods. Finally, given the adjusted prices, we provide a method for estimating the bounds on individual compensating variations that place bounds on the welfare cost of the environmental quality change.

If we have multiple observations of change in environmental quality indicators, we can relate the price adjustments we calculate to these indicators. This could be done for different household types, or household characteristics could be included in the analysis. This would help in the transfer of benefit estimation from one situation and local population mix to another.

Open Access This article is distributed under the terms of the Creative Commons Attribution 4.0 International License (http://creativecommons.org/licenses/by/4.0/), which permits unrestricted use, distribution, and reproduction in any medium, provided you give appropriate credit to the original author(s) and the source, provide a link to the Creative Commons license, and indicate if changes were made.

\section{References}

Adams A, Blundell R, Browning M, Crawford I(2015) Prices versus preferences: taste change and revealed preference. IFS Working Paper W15/11 (2015)

Afriat S (1967) The construction of utility functions from expenditure data. Int Econ Rev 8(1):67-77

Blackorby C, Donaldson D (1993) Adult-equivalence scales and the economic implementation of interpersonal comparisons of well-being. Soc Choice Welfare 10:335-361

Blundell R, Powell J (2007) Censored regression quantiles with endogenous regressors. J Econom 141:65-83 
Blundell R, Browning M, Crawford I (2003) Nonparametric Engel curves and revealed preference. Econometrica 71(1):205-240

Blundell R, Browning M, Crawford I (2008) Best nonparametric bounds on demand responses. Econometrica 76(6):1227-1262

Blundell R, Kristensen D, Matzkin R (2014) Bounding quantile demand functions using revealed preference inequalities. J Econom 117:112-127

Blundell R, Browning M, Cherchye L, Crawford I, De Rock B, Vermeulen F (2015) Sharp for SARP: nonparametric bounds on the behavioural and welfare effects of price changes. Am Econ J Microecon 7(1):43-60

Bockstael N, McConnell K (1993) Public goods as characteristics of non-market commodities. Econ J 103:1244-1257

Chavas JP (1984) The theory of mixed demand functions. Eur Econ Rev 24:321-344

Crooker J, Kling CL (2000) Nonparametric bounds on welfare measures: a new tool for nonmarket valuation. J Environ Econ Manag 39(2):145-161

Erwin Diewert W (1973) Afriat and revealed preference theory. Rev Econ Stud 40(3):419-425

Feenberg D, Mills ES (1980) Measuring the benefits of water pollution abatement. Academic, San Diego, CA

Fisher FM, Shell K (1971) Taste and quality change in the pure theory of the true cost of living index. In: Griliches Z (ed) Price indexes and quality change: studies in new methods of measurement. Harvard University Press, Cambridge

Fostel A, Scarf HE, Todd MJ (2004) Two new proofs of Afriat's theorem. Econ Theor 24:211-219

Gorman WM (1956) A possible procedure for analysing quality differentials in the egg market. London School of Economics, mimeo, reprinted in Rev Econ Stud 47:843-856 (1980)

Herriges JA, Kling CL, Phaneuf DJ (2004) What's the use? Welfare estimates from revealed preference models when weak complementarity doesn't hold. J Environ Econ Manag 47:55-70

Hicks JR (1940) The valuation of the social income. Economica 7:105-124

Houthakker HS (1950) Revealed preference and the utility function. Economica 17:159-174

Imbens G, Newey W (2009) Identification and estimation of triangular simultaneous equations models without additivity. Econometrica 77(5):1481-1512

Koenker R, Bassett G Jr (1978) Regression Quantiles. Econometrica 46(1):33-50

Lancaster K (1966) A new approach to consumer theory. J Polit Econ 74(2):132-157

Lewbel A (1985) A unified approach to incorporating demographic or other effects into demand systems. Rev Econ Stud 52:1-18

Matzkin RL (2007) Heterogeneous choice. In: Blundell R, Newey W, Persson T (eds) Advances in economics and econometrics, theory and applications, Ninth World Congress of the Econometric Society. Cambridge University Press, Persson

Muellbauer J (1975) The cost of living and taste and quality change. J Econ Theory 10:269-283

Neary P, Roberts K (1980) The theory of household behaviour under rationing. Eur Econ Rev 13(1):25-42

Palmquist RB (2005) Weak complementarity, path independence, and the intuition of the Willig condition. J Environ Econ Manag 49(1):103-115

Pollak RA (1989) The theory of the cost-of-living index. Oxford University Press, Oxford

Pollak RA, Wales TJ (1980) Comparison of the quadratic expenditure system and translog demand systems with alternative specifications of demographic effects. Econometrica 48:595-612

Rothbarth E (1941) The measurement of changes in real income under conditions of rationing. Rev Econ Stud 8:100-107

Samuelson PA (1948) Consumption theory in terms of revealed preference. Economica 15:243-253

Smith VK, Banzhaf HS (2004) A diagrammatic exposition of weak complementarity and the Willig condition. Am J Agric Econ 86:455-466

Smith VK, Banzhaf HS (2007) Quality adjusted price indexes and the Willig condition. Econ Lett 94(1):43-48

Smith VK, Evans MF, Banzhaf HS, Poulos C (2010) Can weak substitution be rehabilitated? Environ Resour Econ 45:203-221

Varian H (1982) The nonparametric approach to demand analysis. Econometrica 50(4):945-973

Varian H (1983) Non-parametric tests of consumer behaviour. Rev Econ Stud 50(1):99-110

Varian H (1988) Revealed preference with a subset of goods. J Econ Theory 46(1):179-185

Willig RD (1978) Incremental consumer's surplus and hedonic price adjustment. J Econ Theory 17:227-253 